\title{
Non-Hodgkin Lymphoma in Children with Primary Immunodeficiencies: Clinical Manifestations, Diagnosis, and Management, Belarusian Experience
}

\author{
Alina Fedorova, ${ }^{1}$ Svetlana Sharapova, ${ }^{2}$ Taisia Mikhalevskaya, ${ }^{3}$ Svetlana Aleshkevich, ${ }^{4}$ \\ Inna Proleskovskaya, ${ }^{5}$ Maria Stsegantseva, ${ }^{6}$ Mikhail Belevtsev, ${ }^{7}$ and Olga Aleinikova ${ }^{8}$ \\ ${ }^{1}$ Clinical Research Department, Belarusian Research Center for Pediatric Oncology, Hematology and Immunology, \\ Borovlyany, 223053 Minsk, Belarus \\ ${ }^{2}$ Laboratory of Immunology, Belarusian Research Center for Pediatric Oncology, Hematology and Immunology, \\ Borovlyany, 223053 Minsk, Belarus \\ ${ }^{3}$ Department of Pathology, Belarusian Research Center for Pediatric Oncology, Hematology and Immunology, \\ Borovlyany, 223053 Minsk, Belarus \\ ${ }^{4}$ Consultative and Outpatient Department, Belarusian Research Center for Pediatric Oncology, Hematology and Immunology, \\ Borovlyany, 223053 Minsk, Belarus \\ ${ }^{5}$ Oncohematological Department N2, Belarusian Research Center for Pediatric Oncology, Hematology and Immunology, \\ Borovlyany, 223053 Minsk, Belarus \\ ${ }^{6}$ Laboratory of Genetic Biotechnology, Belarusian Research Center for Pediatric Oncology, Hematology and Immunology, \\ Borovlyany, 223053 Minsk, Belarus \\ ${ }^{7}$ Research Department, Belarusian Research Center for Pediatric Oncology, Hematology and Immunology, \\ Borovlyany, 223053 Minsk, Belarus \\ ${ }^{8}$ Belarusian Research Center for Pediatric Oncology, Hematology and Immunology, Borovlyany, 223053 Minsk, Belarus
}

Correspondence should be addressed to Alina Fedorova; alina_fedorova@list.ru

Received 29 September 2014; Accepted 11 January 2015

Academic Editor: Wolfram Klapper

Copyright (C) 2015 Alina Fedorova et al. This is an open access article distributed under the Creative Commons Attribution License, which permits unrestricted use, distribution, and reproduction in any medium, provided the original work is properly cited.

\begin{abstract}
Introduction. Non-Hodgkin lymphoma (NHL) is the most frequent malignancy associated with primary immune deficiency disease (PID). We aimed to present the clinical characteristics and outcomes of Belarusian children with PID who developed NHL. Procedure. We reviewed 16 patients with PID and NHL. Eight patients had combined PID: 5-Nijmegen breakage syndrome, 1-Bloom syndrome, 1-Wiskott-Aldrich syndrome, and 1-X-linked lymphoproliferative syndrome. Results. In 75\% cases PID was diagnosed simultaneously or after the NHL was confirmed. PID-associated NHL accounted for 5.7\% of all NHL and was characterized by younger median age $(6.3$ versus 10.0 years, $P<0.05)$ and by prevalence of large-cell types $(68.8 \%$ versus $24.5 \%$, $P<0.001)$. Children with combined PID had median age of 1.3 years; 5 of them developed EBV-associated diffuse large Bcell lymphoma with lung involvement. Five of 6 patients with chromosomal breakage syndrome developed T-NHL. Six patients died of infections; two died after tumor progression; one child had early relapse; two died of second NHL and one of secondary hemophagocytic syndrome. Overall, 4 children are alive and disease-free after a follow-up from 1.4 to 5.7 years. Conclusions. PID needs to be diagnosed early. Individualized chemotherapy, comprehensive supportive treatment, and hematopoietic stem cell transplantation may improve survival of children with PID and NHL.
\end{abstract}

\section{Introduction}

Lymphoma is known to be the most frequent malignancy associated with immunodeficiency, either congenital or acquired [1-4]. Advances in diagnosis of primary immune deficiency diseases (PID) and supportive management of infectious complications have led to an increase in life expectancy of children with PID but, also, to the more 
frequent prevalence of non-Hodgkin lymphoma (NHL) as they got older [1]. This makes the choice of treatment strategy a challenging clinical issue. Limited therapy tolerance, infectious status, and immunodeficiency often preclude the standard approach for pediatric NHL treatment.

In this review we aimed to present the clinical characteristics, laboratory data, treatment, and outcomes of 16 children with different types of PID who developed NHL and were diagnosed in our center.

\section{Patients and Methods}

From January, 1996, to January, 2014, the diagnosis of NHL was established in 295 patients up to 18 years of age admitted in Belarusian Research Center for Pediatric Oncology, Hematology and Immunology. Two of them had an HIV-associated NHL, and 16 children suffered from some type of PID. Other 277 patients had no proven immunodeficiency disease and were compared to immunocompromised group in regard to clinical features and outcome.

Histological classification of lymphomas was performed and revised according to World Health Organization classification, 2008 [6]. Immunohistochemistry on conventional paraffin sections and/or immunophenotyping of cell suspensions were performed in 15 cases of PID-associated NHL. Besides the panel of antilymphoid antibodies, monoclonal anti-EBV (Epstein-Barr virus) antibody (LMP CS.14, Dako, USA) was used in 9 cases. Staging was based on criteria of St. Jude staging system [7]. Patients without PID were treated according to modified NHL-BFM90/95 regimen and ALCL99 protocol [5, 8-13]. Before 2001, we used methotrexate infusion $1 \mathrm{~g} / \mathrm{m}^{2} / 36$ hours versus of $5 \mathrm{~g} / \mathrm{m}^{2} / 24$ hours regimen. Since 2004 we used from 2 to 4 infusions of rituximab in standard dose of $375 \mathrm{mg} / \mathrm{m}^{2}$, added to BFM-like chemotherapy courses for B-NHL. Immunocompromised children were treated with full or reduced intensity protocol chemotherapy depending on initial infectious status or with R-EPOCH scheme [14]. Three children with PID received individual treatment, detailed below.

For the same period 230 children were diagnosed as having PID disease, including 17 cases of Nijmegen breakage syndrome (NBS) and 26 cases of different types of combined PID. Sixteen patients with PID, who developed NHL, were compared with other registered PID patients.

Diagnosis of PID disease was based on diagnostic criteria of PID of the last classification from the International Union of Immunological Societies Expert Committee for Primary Immunodeficiency, 2013 [15]. Immunogram was used to reveal PID as well as genetic investigations. Serum concentrations of immunoglobulins IgG, IgA, and IgM were measured by nephelometry. Relative and absolute numbers of peripheral blood T- and B-lymphocytes and NK-cells were calculated by flow-cytometry using monoclonal antibodies (Becton Dickinson, USA). Mutational analysis of PID genes was performed; NBS1, WAS, RAG1, RAG2, ADA, IL7Ra, IL2Ra, Jak3, SH2D1A, and DNA ligase IV genes were sequenced. Database for evaluation the genes variants and construct primers used Ensembl Genome Browser. The number of TRECs and KRECs was determined by RT-qPCR on the Step One Plus (Applied Biosystems, USA). Combined (severe +/-) immunodeficiency with non defined genetic basis was diagnosed if reduced TREC level was determined in DNA material (bone marrow or blood samples/smears) taken before initiation of treatment. Chromosomal instability analysis was performed to reveal high chromosomal breakage in lymphocytes. Chromosomal somatic mutations were revealed by conventional G-banding.

Statistical data on disease cases were provided by Childhood Cancer Subregistry of Belarus (the member of International Association of Cancer Registry) and by National Children Registry of PID Diseases.

Parents of all patients gave written informed consent for NHL treatment according to protocols approved by the local Ethical Committee as well as for blood sampling and processing for PID diagnosis.

Analysis of overall survival (OS) was performed using the Kaplan Meier method, with differences compared by the log-rank test. Date of analysis was September 1, 2014. Fisher and Mann-Whitney tests were used to evaluate differences in distribution of individual parameters in subsets of patients.

\section{Results}

Patients with PID-associated NHL accounted for 5.7\% of all registered cases of NHL. Patient characteristics and clinical data are presented in Tables 1 and 2. There were 10 boys and 6 girls. Median age at NHL diagnosis was younger in children with PID (6.3 years, range $0.7-15.9$ years) compared to immunocompetent patients (10.0 years, range 1.3-17.9 years) $(P<0.05)$. Distribution of histologic types in morphological spectrum of NHL was different in patients with PID in comparison with immunocompetent patients with the prevalence of large-cell types (68.8\% versus $23.5 \%$, $P<0.001)$ including cases of NHL, which are typical for adults. All patients except one had an advanced stage of disease at the time of diagnosis, which is significantly more often compared to immunocompetent cohort ( $93.8 \%$ versus $72.2 \%, P<0.05)$.

In 12 of 16 cases, PID was diagnosed simultaneously or after the NHL was confirmed, including 6 cases of postmortem diagnosis. Inclusion criteria to perform immunological and molecular tests for PID were very young age at diagnosis of NHL, unusual NHL type for the certain age group, constitutional abnormalities, history of severe recurrent infections or family history of sibling's death due to infections or inborn abnormalities, and thymic pathology found at autopsy. If immunological and/or genetic data did not confirm PID in HIV-negative patient, he was considered as immunocompetent and eligible for comparative analysis.

Six out of 16 patients suffered chromosomal breakage syndrome associated with immunodeficiency (Tables 2 and 3). NBS was found in 5 children (mutation 657del5 in NBS1 gene typical for Slavic population was found in all cases) and Bloom syndrome was found in one patient. Median age at diagnosis of NHL in these patients was 12.5 years, which is similar enough to immunocompetent cohort. 
TABLE 1: Characteristics of patients with $\mathrm{NHL}^{\mathrm{a}}$.

\begin{tabular}{|c|c|c|}
\hline Characteristic & $\begin{array}{l}\text { Patients with } \text { PID }^{\mathrm{b}} \text {, } \\
n=16(\%)\end{array}$ & $\begin{array}{c}\text { Immunocompetent } \\
\text { patients, } n=277 \\
(\%)\end{array}$ \\
\hline \multicolumn{3}{|l|}{ Sex } \\
\hline $\mathrm{M}$ & $10(62.5)$ & $220(79.2)$ \\
\hline $\mathrm{F}$ & $6(37.5)$ & $57(20.8)$ \\
\hline \multicolumn{3}{|l|}{ Age } \\
\hline $0-4$ & $6(37.5)$ & $52(18.8)$ \\
\hline $5-9$ & $5(31.25)$ & $85(30.7)$ \\
\hline $10-14$ & $2(12.5)$ & $86(31.0)$ \\
\hline$\geq 15$ & $3(18.75)$ & $54(19.5)$ \\
\hline \multicolumn{3}{|l|}{ Stage } \\
\hline $\mathrm{I}+\mathrm{II}$ & $1(6.25)$ & $77(27.8)$ \\
\hline III + IV & $15(93.75)$ & $200(72.2)$ \\
\hline \multicolumn{3}{|l|}{ Morphology } \\
\hline Burkitt & $2(12.5)$ & $135(48.7)$ \\
\hline T-lymphoblastic & $3(18.75)$ & $52(18.8)$ \\
\hline B-lymphoblastic & 0 & $19(6.9)$ \\
\hline $\mathrm{DLBCL}^{\mathrm{c}}$ & $6(37.5)$ & $16(5.8)$ \\
\hline $\mathrm{PMLBCL}^{\mathrm{d}}$ & 0 & $14(5.1)$ \\
\hline $\mathrm{ALCL}^{\mathrm{e}}$ systemic & $2(12.5)$ & $33(11.9)$ \\
\hline ALCL cutaneous & $1(6.25)$ & 0 \\
\hline $\mathrm{PTCL}^{\mathrm{f}}$ & $2(12.5)$ & $2(0.7)$ \\
\hline B-NHL, unspecified & 0 & $6(2.2)$ \\
\hline \multicolumn{3}{|l|}{ Immunological lineage } \\
\hline $\mathrm{B}$ & $8(50.0)$ & $190(68.6)$ \\
\hline $\mathrm{T}$ & $8(50.0)$ & $87(31.4)$ \\
\hline
\end{tabular}

${ }^{\mathrm{a}}$ Non-Hodgkin lymphoma, ${ }^{\mathrm{b}}$ primary immune deficiency disease, ${ }^{\mathrm{c}}$ diffuse large B-cell lymphoma, ${ }^{\mathrm{d}}$ primary mediastinal large B-cell lymphoma, ${ }^{e}$ anaplastic large cell lymphoma, and ${ }^{\mathrm{f}}$ peripheral T-cell lymphoma.

T-cell NHL dominated (found in 5 out of 6 children) and was T-mature in most cases. Just in one case lymphoma cells had immunophenotype of immature cortical T-cells. Despite full dosage of chemotherapy, complete remission (CR) was achieved only in $50 \%$ cases (in 3 out of 6 ) and progression was observed in 1 patient. Every child with chromosomal breakage syndrome had severe infectious outbreaks during induction therapy, which happened to be fatal complications for two children with unrecognized NBS. One girl developed the second lymphoma 4.5 years after the first NHL, and one boy developed secondary hemophagocytic syndrome 7 months after intensive chemotherapy was completed.

Out of total 17 NBS patients registered in the National Children Registry of PID Diseases lymphoproliferative disorders occurred in $9(52.9 \%)$ patients ranging from 4 to 21 years. Except 5 children with NHL included to analyzing group, we observed 4 patients with acute lymphoblastic leukemia (of T-cell differentiation in 3 cases: pro-T in 2 and T-mature in 1). Age of other observed NBS patients without malignancies ranged from 5 months to 20 years. Unfortunately there is no data of any living older people with NBS in Belarus.
There were 8 children with different types of combined PID. These patients were the youngest of the whole group with median age of 1.3 years. Six of 8 children had very aggressive large cell lymphomas with focal pulmonary lesions: 5 had diffuse large B-cell lymphoma (DLBCL) and 1 had peripheral T-cell lymphoma. Five cases were tested for EBV infection and in all cases EBV-associated lymphoma was confirmed by immunohistochemistry. Four children had symptoms of generalized EBV infection at the time of NHL diagnosis. Management of those patients was complicated by very young age, low weight (from 4.5 to $9.3 \mathrm{~kg}$ in represented cohort of children with high grade large cell lymphomas), multiple previous infectious episodes, and severity of EBV infection, which seemed to be hardly possible to cure after NHL development. Reduced intensity chemotherapy regimens were used in this group. Complete clinical recovery was achieved only in one case of 7-month-old girl, who had no symptoms of active EBV infection at the time of initiation of chemotherapy (Tables 2 and 3, patient number 1). All other died of infectious complications without CR achievement.

NHL developed in $30.8 \%$ of children (in 8 out of 26) with combined PID registered in PID database. Currently there are only 4 children under our observation ranging from 1.9 to 11.8 years of age, who did not undergo hematopoietic stem cell transplantation (HSCT) and have no signs of malignant disorders.

We observed malignant transformation and DLBCL development in a 10-month-old boy with combined PID and generalized EBV infection (Tables 2 and 3, patient number $6)$. The time interval between appearance of the first signs of EBV infection and appearance of pulmonary tumor lesions was 2.5 months. The patient died after the first course of chemotherapy (R-EPOCH with $25 \%$ reduction of all agents) due to the sepsis, EBV-infection, and multiple organ failure.

We also observed a case of abdominal Burkitt lymphoma in a 6-year-old boy with X-linked lymphoproliferative syndrome (XLPS-1) without regard to EBV infection (Tables 2 and 3, patient number 16). Patient had no family or infectious anamnesis and tolerated chemotherapy well. Lymphoma cells were EBV-negative by immunohistochemistry. His PID was recognized only a year later after NHL diagnosis. Absence of IgG serum level recovery within a year after the chemotherapy treatment combined with rituximab, while the level of CD19 and CD20 positive cells became normal within six months after the end of treatment, was the reason to perform genetic investigations and mutation in SH2D1A gene on Xq25 was found. The parents of the child refused alloHSCT. Patient monthly receives intravenous immunoglobulin and has no evidence of lymphoma or severe viral infections with a follow-up of 5.7 years.

The treatment details are given in Table 2. One girl with primary cutaneous ALCL, anaplastic lymphoma kinase (ALK) negative, with localized involvement of the eye lid received only brachytherapy. Two children with combined PID received only dexamethasone, both of them initially had leucopenia, agranulocytosis, and generalized infection, mixed EBV and bacterial, and both died few days after admission. Other 13 patients received at least one course of chemotherapy. The individually administered therapy was 


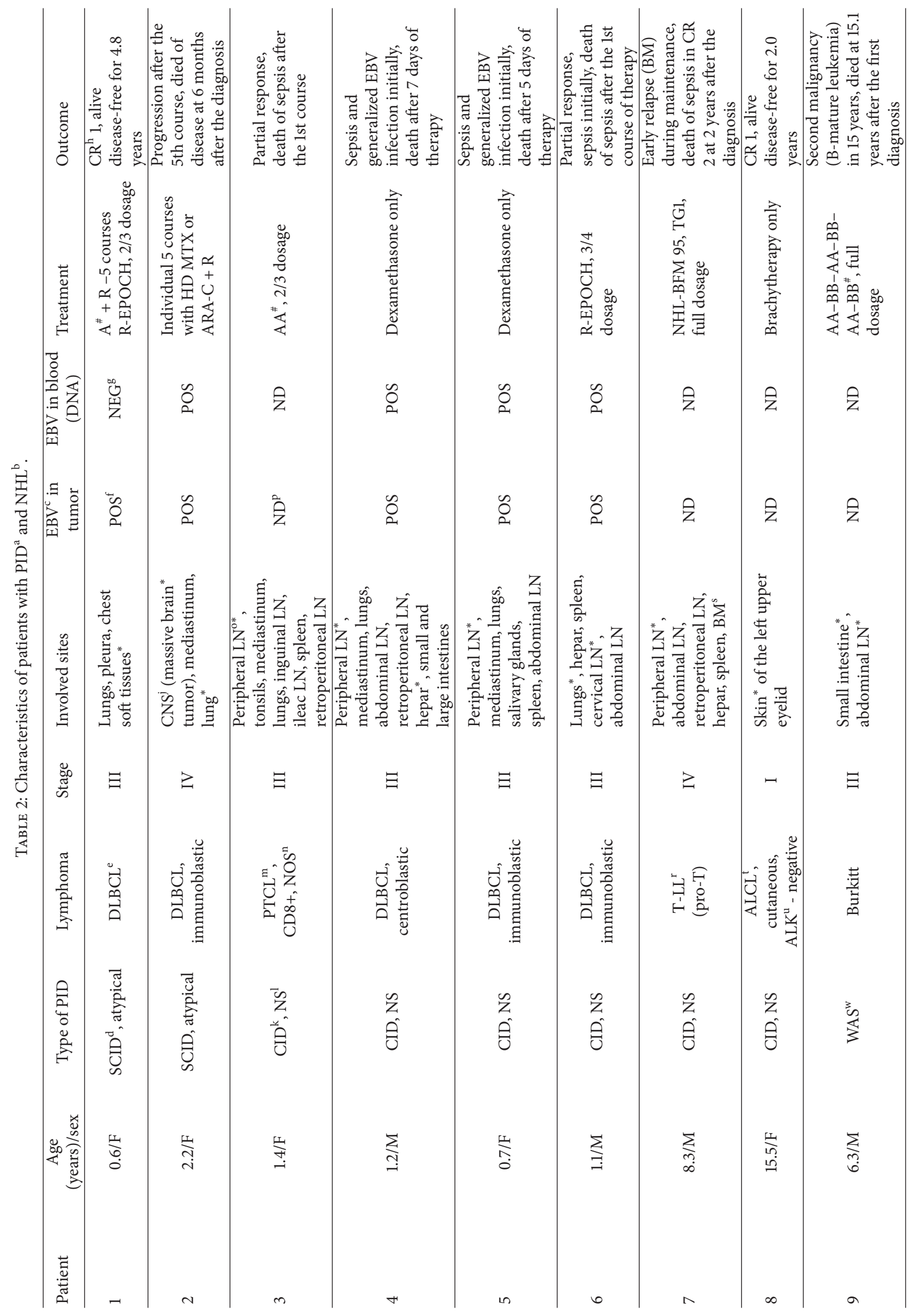




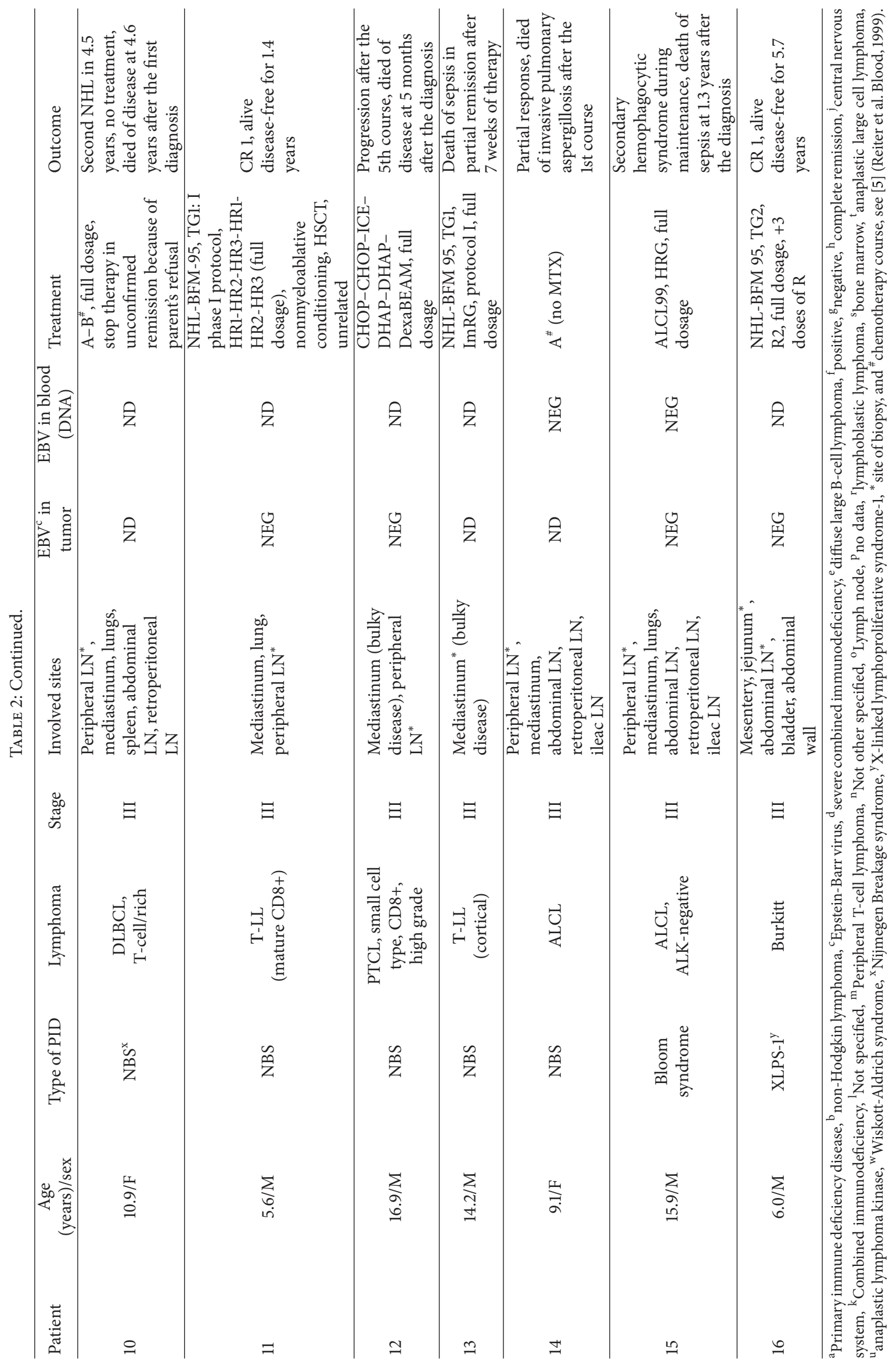




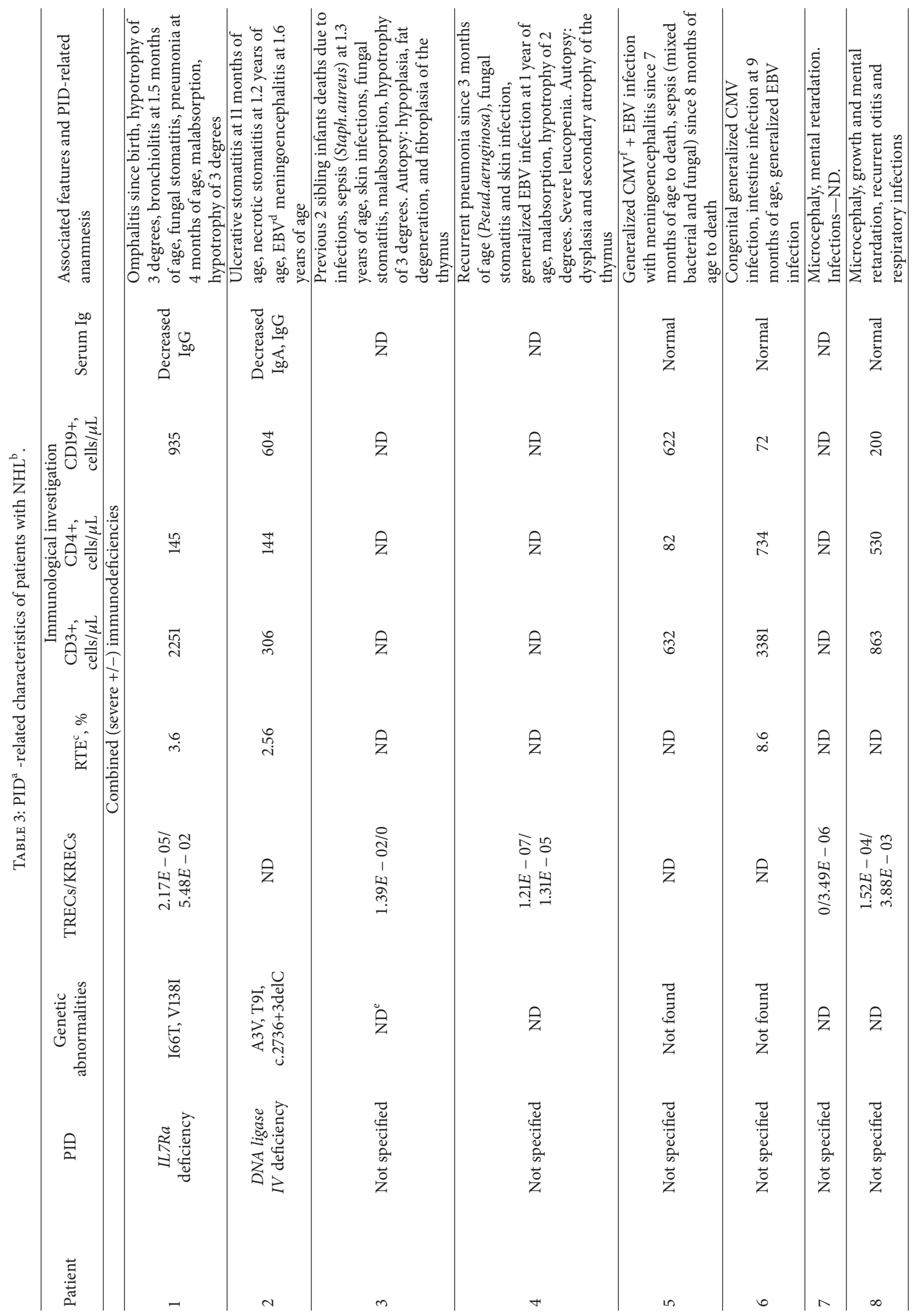




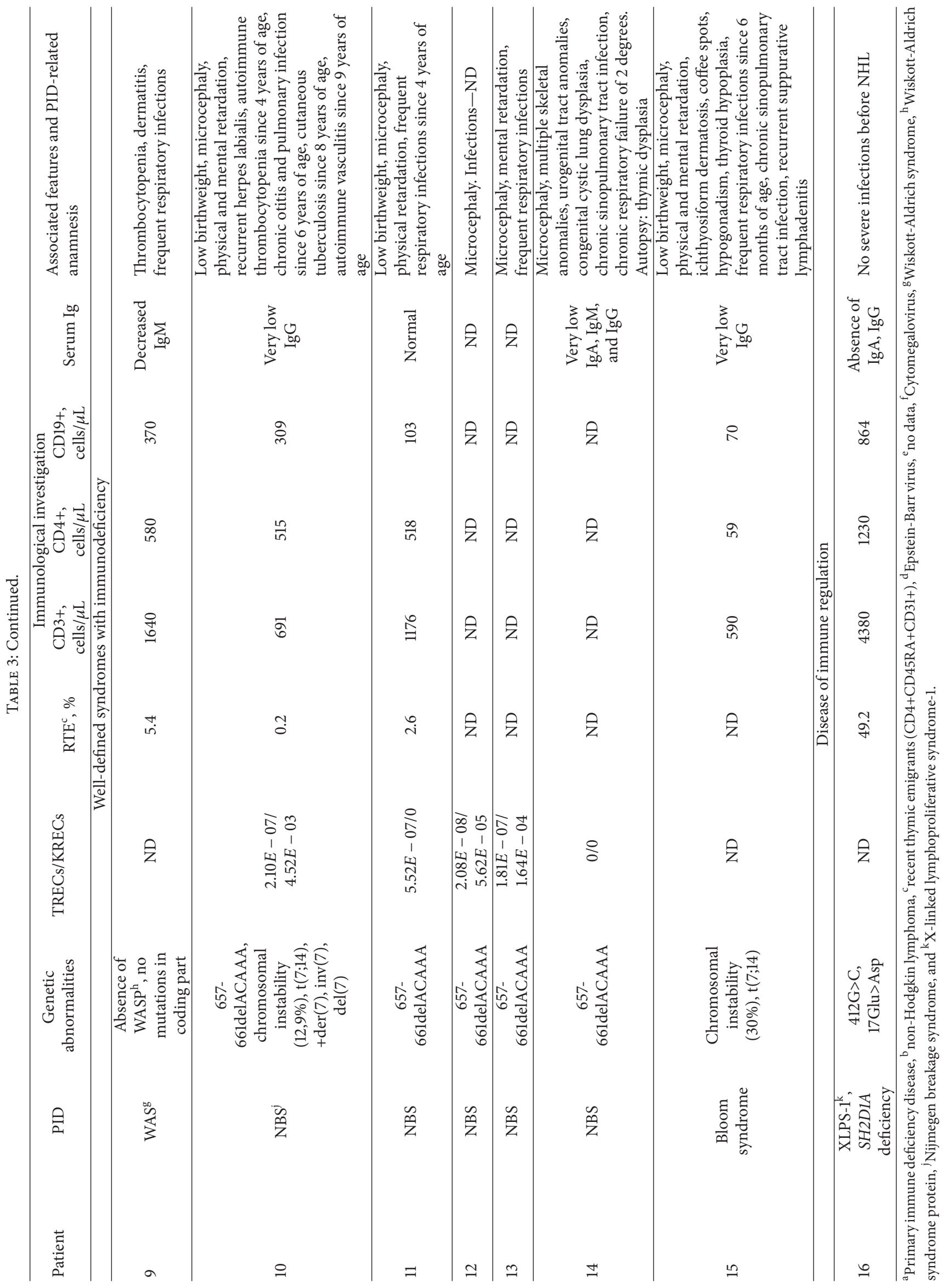


TABLE 4: Treatment complications in first line therapy (15 patients, 51 courses/element of chemotherapy).

\begin{tabular}{lc}
\hline Complication & Number of episodes \\
\hline Organ failure caused by sepsis & 4 \\
Myelosuppression (grade 3-4) & 49 \\
Febrile neutropenia & 19 \\
Sepsis & 7 (7 positive cultures) \\
Stomatitis (grade 3-4) & 7 \\
Necrotic skin ulcers & 2 \\
Cellulitis & 2 \\
Systemic vasculitis & 1 \\
Hepatotoxicity (grade 3-4) & 2 \\
Pancreatitis & 4 \\
Steroid-induced diabetes & 1 \\
Gastrointestinal hemorrhage & 5 \\
Enterocolitis & 2 \\
Sinusitis & 1 \\
Pneumonia & 3 \\
Pulmonary aspergillosis & 2 \\
Pulmonary hemorrhage & 2 \\
Thrombosis & 2 \\
Neurotoxicity (grade 2) & 2 \\
Cytomegalovirus infection & 4 \\
Epstein-Barr virus infection & 3 \\
\hline
\end{tabular}

given to a girl with massive central nervous system involvement and included high dose methotrexate or cytarabine, dexamethasone, and rituximab intravenous and intrathecal, with or without other block elements, but rapid brain tumor growth occurred after the fifth course of chemotherapy while EBV DNA by PCR remained positive in either blood or cerebrospinal fluid (Tables 2 and 3 , patient number 2).

All patients on chemotherapy experienced grade 3 and 4 complications (Table 4). The most common were neutropenic infections and hemostatic disorders. There were 6 deaths related to first line treatment, 5 from sepsis and 1 from invasive aspergillosis with massive pulmonary bleeding.

During the treatment of PID-associated NHL CR was achieved only in $50 \%$ of cases. Two patients died after early tumor progression during the intensive therapy, and one child relapsed while on maintenance therapy. We observed second lymphoproliferative disorder in two patients and secondary hemophagocytic syndrome in one case. Overall, 4 (25\%) children with PID are alive and disease-free with follow-up ranging from 1.4 to 5.7 years. The probability of 10 -year OS for children with PID was $0.23 \pm 0.13$, which is dramatically worse than the survival of immunocompetent patients with NHL (OS $0.81 \pm 0.02, P<0.00001)$ in our country.

\section{Discussion}

PID disease is known to be the strong risk factor for developing a malignancy in childhood. NHL account for more than $60 \%$ of the tumors in children with PID and develop in 2$4 \%$ of patients at the age before 18 years. Lymphoma type and frequency varies among children with different types of PID. The highest, 250-fold risk, have patients with chromosomal breakage syndromes [1-4]. We observed 5.7\% frequency of PID-associated NHL among children with NHL, which is higher than was previously reported $[1,16,17]$. Over $90 \%$ of Belarusian pediatric patients with NHL received treatment in our center which makes our data be representative for our region with population more than ten million. In our series of patients the diagnosis of NHL preceded the diagnosis of PID disease in $75 \%$ cases, even in children with NBS, who has typical features of dysmorphogenesis. In addition to progressing microcephaly, low birthweight, growth retardation, and characteristic bird-like facial features, 2 children with NBS had multiple congenital abnormalities of skeletal and/or urogenital system but were still not recognized as having PID before malignant lymphoma developed. Likewise, 4 from 8 children with different combined PID were not recognized as having immunodeficiency before NHL developed, in spite of history of severe infections in the past. Their immune status was, therefore, not tested and preventive measures were not complied and did not receive intravenous immunoglobulin. As a result, their initial infectious status was supposed to be worse and made their management more challenging. Survival of children with PID-associated NHL is much lower in comparison with immunocompetent patients in all reports and ranges from 35 to 58\% [16-21]. The outcome of our patients was poor with only 4 survived out of 16 children. We consider late recognition of PID was the main reason of our bad outcomes. Attention and awareness of pediatricians can contribute to earlier immunological and molecular genetic testing to children with suspected PID to reveal those who need to perform alloHSCT early to restore immune system and to provide special management to all children with PID keeping in mind they are at higher risk of malignancy development.

Type of PID-associated NHL is very different with the prevalence of typical for adults histology. Each type of immunodeficiency has its own risk factors and is associated with a particular type of lymphoma [3, 16-20, 22]. DLBCL is considered to be the most common type, frequently associated with EBV and having a predilection for extranodal sites. We received similar data and observed series of DLBCL accounted for $37.5 \%$ of NHL. All DLBCL cases were EBVassociated and characterized by rather massive lung involvement.

DLBCL was predominant variant of NHL, which was occurred in young children with combined PID. Multiple pulmonary lesions may also manifest an extensive fungal infection or tuberculosis. We recommend performing lung biopsy early after the detection of pulmonary foci in order not to lose time on preventive antibacterial or antifungal treatment in patients with combined PID and documented EBV infection. We also observed a case of malignant transformation of EBV-associated lymphadenitis resistant to antiviral treatment. EBV infection is a potentially premalignant event in children with PID $[23,24]$. We are convinced that the EBV prophylaxis, early aggressive antiviral treatment, and viral load monitoring in a case of clinical manifestation of EBV infection increase their chance to survive. 
In the present group of patients 4 out of 5 patients with NBS were suffering from T-cell lymphomas. However, both $\mathrm{T}$ - and B-cell lymphomas were described in literature with some prevalence of B-cell lineage [19-22]. But still remains unknown whether development of T-cell or B-cell NHL depends more on defect and degree of immunodeficiency rather than on chromosomal instability in NBS patients.

Complication rate during the treatment of NHL was high in our cohort of children with combined PID and in some patients with chromosomal breakage syndrome which is consistent with other reports [17-20]. Tolerability of chemotherapy was limited mostly by infectious status rather than toxic side effects. Patients with combined PID and NBS had very low mRNA TREC and KREC expression which explained their predisposition to both bacterial and viral infections (Table 3). Therefore standard pediatric NHL regimens proved to be very toxic for these patients. Modified chemotherapy schemes combined with monoclonal antibodies should be designed for the treatment of PID-associated NHL.

Clinically, alloHSCT has been used successfully for lymphomas arising in XLPS, combined PID, WAS, X-linked hyper-IgM syndrome, and even NBS [16, 25-28]. Based on our observations of developing of second lymphoproliferative malignancies in the future in children with PID, we consider that alloHSCT should be done in survived patients in first remission with any type of PID who have severe defects of immune function.

\section{Conclusion}

Our data shows that PID disease needs to be diagnosed as early as possible and to be corrected with transplantation or appropriate management to prevent severe and recurrent infections and foremost EBV infection. These patients are at a very high risk to develop NHL of both $\mathrm{T}$ and $\mathrm{B}$ lineage, but of those NHL types, which are mostly typical for adults. Unrecognized PID before NHL diagnosis and infectious complications during chemotherapy were the main reasons of poor outcome of our patients with PID-associated NHL. Early PID diagnosis, individualized chemotherapy, comprehensive supportive treatment, and alloHSCT performance may improve their survival.

\section{Conflict of Interests}

The authors declare that there is no conflict of interests regarding the publication of this paper.

\section{References}

[1] J. Chinen, D. Anmuth, A. R. K. Franklin, and W. T. Shearer, "Long-term follow-up of patients with primary immunodeficiencies," Journal of Allergy and Clinical Immunology, vol. 120, no. 4, pp. 795-797, 2007.

[2] N. F. C. C. de Miranda, A. Björkman, and Q. PanHammarström, "DNA repair: the link between primary immunodeficiency and cancer," Annals of the New York Academy of Sciences, vol. 1246, no. 1, pp. 50-63, 2011.
[3] H. Tran, J. Nourse, S. Hall, M. Green, L. Griffiths, and M. K. Gandhi, "Immunodeficiency-associated lymphomas," Blood Reviews, vol. 22, no. 5, pp. 261-281, 2008.

[4] S. Oertel and H. Reiss, Immunosurveillance, Immunodeficiency and Lymphoproliferations, Springer, Heidelberg, Germany, 2002.

[5] A. Reiter, M. Schrappe, M. Tiemann et al., "Improved treatment results in childhood B-cell neoplasms with tailored intensification of therapy: a report of the Berlin-Frankfurt-Munster Group Trial NHL-BFM-90," Blood, vol. 94, no. 10, pp. 3294-3306, 1999.

[6] S. H. Swerdlow, E. Campo, N. L. Harris et al., WHO Classification of Tumours of Haematopoietic and Lymphoid Tissues, IARC Press, Lyon, France, 4th edition, 2008.

[7] S. B. Murphy, "Classification, staging and end results of treatment of childhood non-Hodgkin's lymphomas: dissimilarities from lymphomas in adults," Seminars in Oncology, vol. 7, no. 3, pp. 332-339, 1980.

[8] A. Reiter, M. Schrappe, W.-D. Ludwig et al., "Intensive ALLtype therapy without local radiotherapy provides a $90 \%$ eventfree survival for children with T-cell lymphoblastic lymphoma: a BFM Group report," Blood, vol. 95, no. 2, pp. 416-421, 2000.

[9] B. Burkhardt, W. Woessmann, M. Zimmermann et al., "Impact of cranial radiotherapy on central nervous system prophylaxis in children and adolescents with central nervous systemnegative stage III or IV lymphoblastic lymphoma," Journal of Clinical Oncology, vol. 24, no. 3, pp. 491-499, 2006.

[10] W. Woessmann, K. Seidemann, G. Mann et al., "The impact of the methotrexate administration schedule and dose in the treatment of children and adolescents with B-cell neoplasms-a report of the BFM Group Study NHL-BFM95," Blood, vol. 105, no. 3, pp. 948-958, 2005.

[11] K. Seidemann, M. Tiemann, M. Schrappe et al., "Short-pulse B-non-Hodgkin lymphoma-type chemotherapy is efficacious treatment for pediatric anaplastic large cell lymphoma: a report of the Berlin-Frankfurt-Münster Group Trial NHL-BFM 90," Blood, vol. 97, no. 12, pp. 3699-3706, 2001.

[12] M.-C. Le Deley, A. Rosolen, D. M. Williams et al., "Vinblastine in children and adolescents with high-risk anaplastic largecell lymphoma: results of the randomized ALCL99-vinblastine trial," Journal of Clinical Oncology, vol. 28, no. 25, pp. 3987-3993, 2010.

[13] L. Brugières, M.-C. le Deley, A. Rosolen et al., "Impact of the methotrexate administration dose on the need for intrathecal treatment in children and adolescents with anaplastic large-cell lymphoma: results of a randomized trial of the EICNHL Group," Journal of Clinical Oncology, vol. 27, no. 6, pp. 897-903, 2009.

[14] J. A. Sparano, J. Y. Lee, L. D. Kaplan et al., "Rituximab plus concurrent infusional EPOCH chemotherapy is highly effective in HIV-associated B-cell non-Hodgkin lymphoma," Blood, vol. 115, no. 15, pp. 3008-3016, 2010.

[15] W. Al-Herz, A. Bousfiha, J.-L. Casanova et al., "Primary immunodeficiency diseases: an update on the classification from the International Union of immunological societies expert committee for primary immunodeficiency," Frontiers in Immunology, vol. 5, article 162, 2014.

[16] C. Booth, K. C. Gilmour, P. Veys et al., "X-linked lymphoproliferative disease due to SAP/SH2D1A deficiency: a multicenter study on the manifestations, management and outcome of the disease," Blood, vol. 117, pp. 53-62, 2011. 
[17] K. Seidemann, M. Tiemann, and G. Henze, “Therapy for nonHodgkin lymphoma in children with primary immunodeficiency: analysis of 19 patients from the BFM trials," Medical and Pediatric Oncology, vol. 33, no. 6, pp. 536-544, 1999.

[18] C. Sandoval and M. Swift, "Treatment of lymphoid malignancies in patients with ataxia-telangiectasia," Medical and Pediatric Oncology, vol. 31, no. 6, pp. 491-497, 1998.

[19] B. Dembowska-Baginska, D. Perek, A. Brozyna et al., "NonHodgkin lymphoma (NHL) in children with Nijmegen Breakage syndrome (NBS)," Pediatric Blood and Cancer, vol. 52, no. 2, pp. 186-190, 2009.

[20] K. Bienemann, B. Burkhardt, S. Modlich et al., "Promising therapy results for lymphoid malignancies in children with chromosomal breakage syndromes (Ataxia teleangiectasia or Nijmegen-breakage syndrome): a retrospective survey," British Journal of Haematology, vol. 155, no. 4, pp. 468-476, 2011.

[21] J. T. Sandlund, M. M. Hudson, W. Kennedy, M. Onciu, and M. B. Kastan, "Pilot study of modified LMB-based therapy for children with ataxia-telangiectasia and advanced stage high grade mature B-cell malignancies," Pediatric Blood and Cancer, vol. 61, no. 2, pp. 360-362, 2014.

[22] M. Gładkowska-Dura, K. Dzierzanowska-Fangrat, W. T. Dura et al., "Unique morphological spectrum of lymphomas in Nijmegen breakage syndrome (NBS) patients with high frequency of consecutive lymphoma formation," The Journal of Pathology, vol. 216, no. 3, pp. 337-344, 2008.

[23] M. L. Gulley, C.-L. Chen, and N. Raab-Traub, "Epstein-Barr virus-related lymphomagenesis in a child with Wiskott-Aldrich syndrome," Hematological Oncology, vol. 11, no. 3, pp. 139-145, 1993.

[24] L. Dupré, G. Andolfi, S. G. Tangye et al., "SAP controls the cytolytic activity of $\mathrm{CD}^{+}$T cells against EBV-infected cells," Blood, vol. 105, no. 11, pp. 4383-4389, 2005.

[25] J. Bagley, M. L. Cortes, X. O. Breakefield, and J. Iacomini, "Bone marrow transplantation restores immune system function and prevents lymphoma in Atm-deficient mice," Blood, vol. 104, no. 2, pp. 572-578, 2004.

[26] K. Kawa, T. Okamura, M. Yasui, E. Sato, and M. Inoue, "Allogeneic hematopoietic stem cell transplantation for EpsteinBarr virus-associated T/NK-cell lymphoproliferative disease," Critical Reviews in Oncology/Hematology, vol. 44, no. 3, pp. 251257, 2002.

[27] L. L. Williams, C. M. Rooney, M. E. Conley, M. K. Brenner, R. A. Krance, and H. E. Heslop, "Correction of Duncan's syndrome by allogeneic bone marrow transplantation," The Lancet, vol. 342, no. 8871, pp. 587-588, 1993.

[28] M. H. Albert, A. R. Gennery, J. Greil et al., "Successful SCT for Nijmegen breakage syndrome," Bone Marrow Transplantation, vol. 45 , no. 4 , pp. $622-626,2010$. 


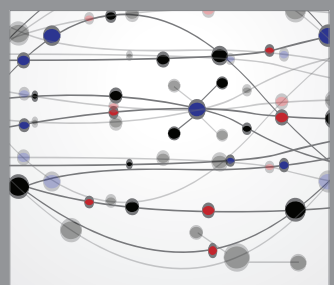

The Scientific World Journal
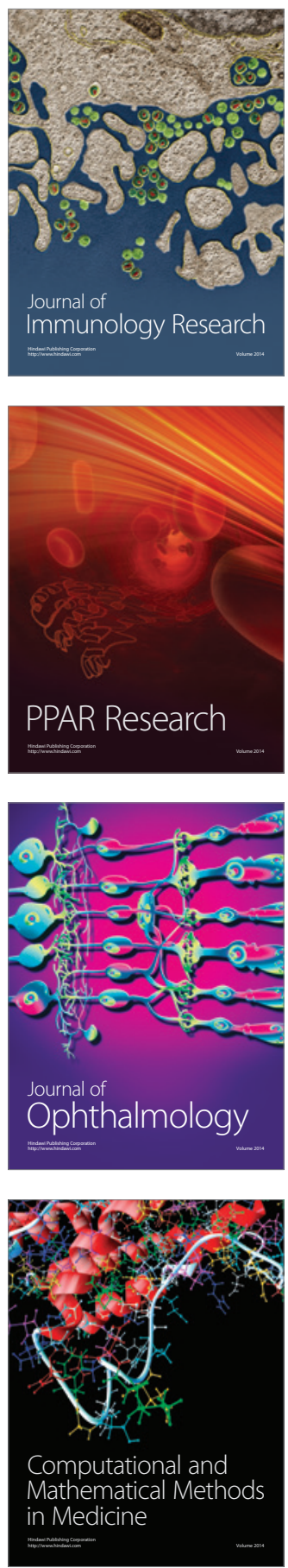

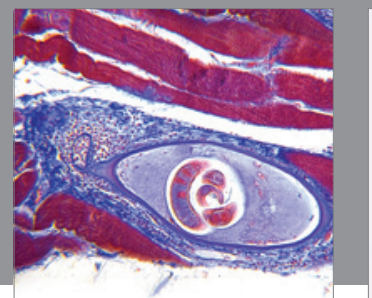

Gastroenterology

Research and Practice
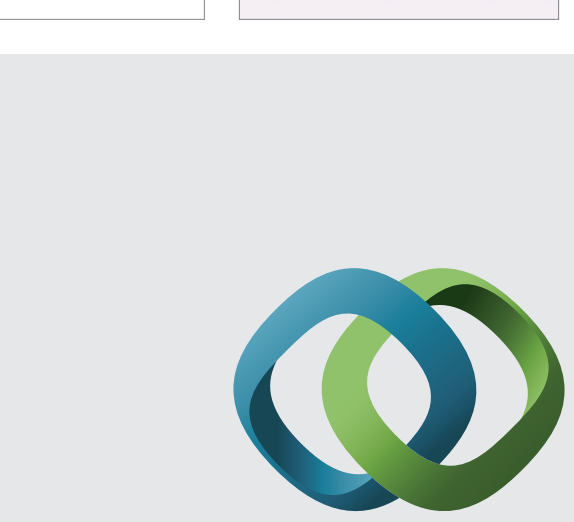

\section{Hindawi}

Submit your manuscripts at

http://www.hindawi.com
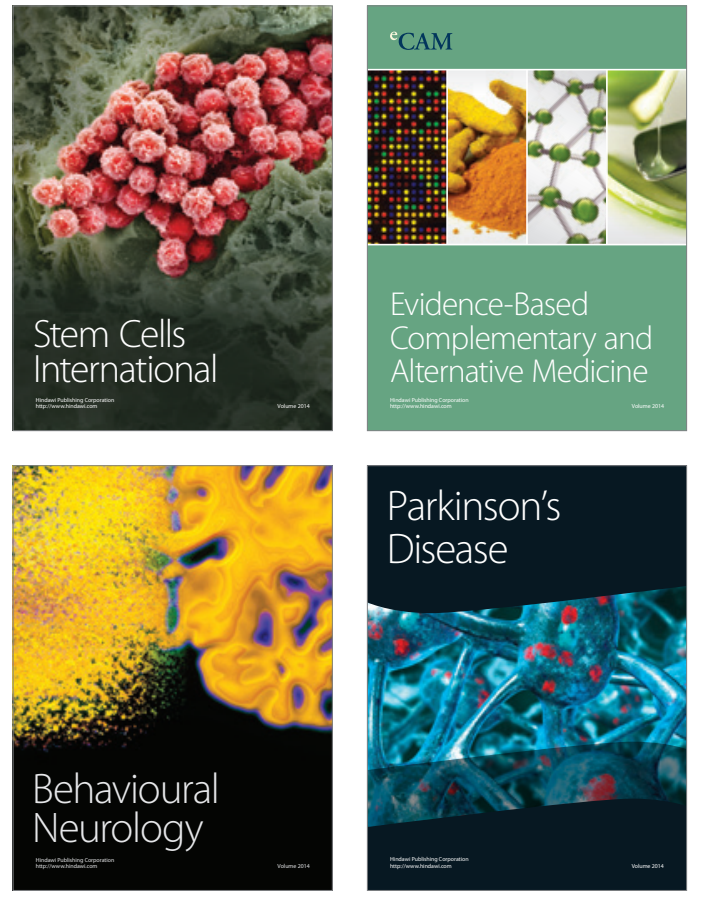
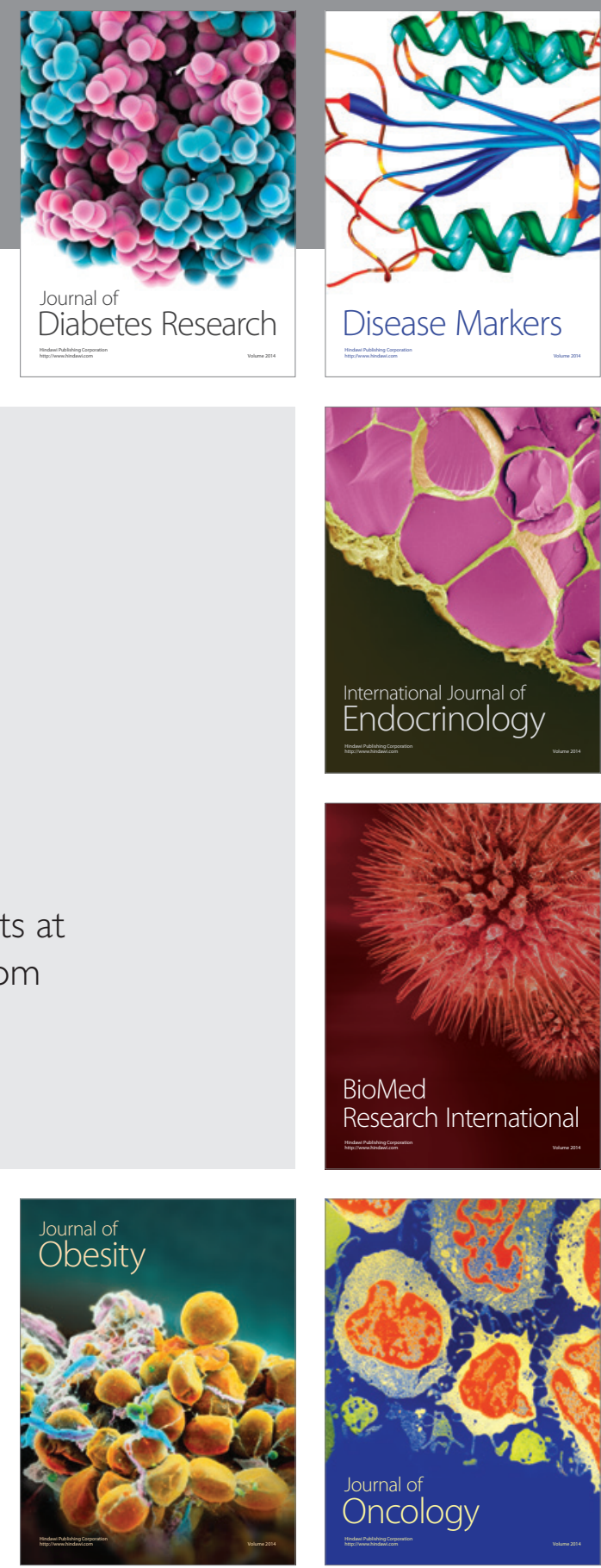

Disease Markers
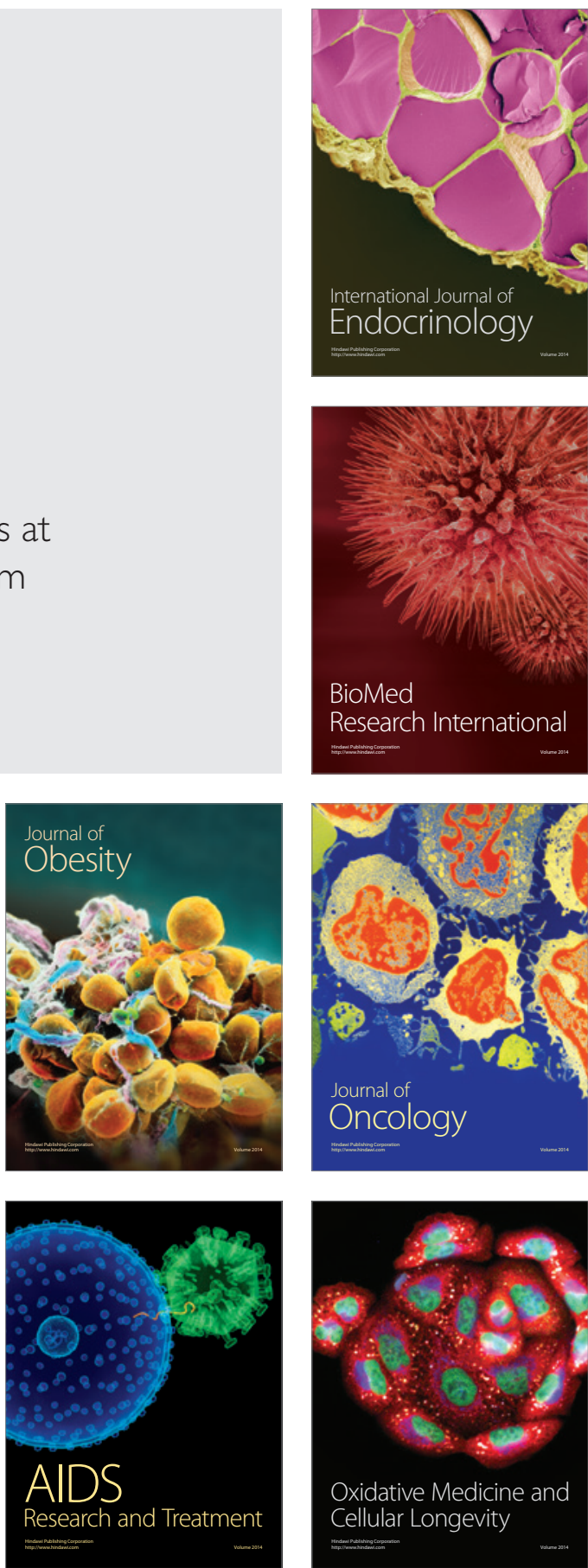